\title{
Speciation of Candida Isolated among Immunocompromised Patients
}

\author{
S. Meenakshi ${ }^{1}$ and R. Vidhya $\operatorname{Rani}^{2} *$ \\ ${ }^{1}$ Department of Microbiology, Madras Medical College, Chennai, Tamilnadu, India \\ ${ }^{2}$ Department of Microbiology Govt Mohankumaramangalam Medical College, Salem, \\ Tamilnadu, India \\ *Corresponding author:
}

\section{A B S T R A C T}

\section{Keywords}

Candida, Immuno-

compromised

Patients

Article Info

Accepted:

12 February 2019

Available Online:

10 March 2019
Immune-compromised patients have lead to rise in infections caused by Candida albicans and by Non Candida albicans. This study was done with 368 samples from 300 immunocompromised patients. The most common species isolated was $C$. albicans (42\%), followed by $C$. tropicalis $(35 \%), C$. pseudotropicalis (5\%), C. krusei (5\%), C. glabarata (4\%), C. parapsilosis (3\%), C. guilliermondii (2\%). CHROM agar candida medium was used in identification of Candida species. It is very important to identify these pathogens at the species level from all the clinical specimens received at laboratory for effective treatment.

\section{Introduction}

Fungal infections involving yeast isolates, especially Candida species are increasingly being identified as etiological agents in nosocomial infections. Despite the clinical importance, the ability to prevent, diagnose and treat Candida infections is difficult particularly in immunocompromised patients (Lata R. Patel et al., 2012). Certain factors that contribute to colonization leading onto infection include prolonged treatment with multiple antimicrobial agents, use of indwelling devices, prolonged neutropenia with cancer, congenital or acquired immunodeficiency, malnutrition, diabetes mellitus, neutropenic ulcers (Manchanda et al., 2011). The incidence of Candida infection is increasing because of a rising number of immunocompromised patients and widespread use of broad spectrum antibiotics. Although Candida albicans remains the most frequently isolated pathogen, other Candida species such as C. tropicalis, C. glabarata, $C$. guilliermondi and $C$. krusei are emerging as opportunistic pathogens (Gugnani et al., 2003). The present study was done to study the prevalence of Candida species and for the speciation of Candida isolates isolated from immunocompromised patients. 


\section{Materials and Methods}

The study was done at madras Medical College Chennai for a period of one year involving 300 immunocompromised patients which included 1. HIV infected persons, 2. Renal transplant patients, 3. Patients suffering from diabetes mellitus, 4. Patients on immunosuppressive therapy especially corticosteroids, 5. Cancer patients and those receiving anticancer therapy 6 . Patients with chronic diseases like TB, COPD or on prolonged antibiotic therapy. The samples were collected according to the clinical presentation: Oral scrapings, Urine, Sputum / tracheal aspirate/bronchial wash, Blood, Vaginal swab, Nasal Swab, CSF, swab from palatal perforation and pus. The samples were inoculated into Sabouraud Dextrose agar supplemented with antibiotics (Chloramphenicol, Gentamicin and tetracycline) to prevent bacterial overgrowth. Cultures are incubated at $28 \mathrm{C}$ and $37 \mathrm{C}$ colonies become apparent within 2-3 days but in some cases as early as 24 hours. Candida Speciation done using CHROMagar which uses chromogenic substrates to react with specific enzymes of the different species resulting in the formation of differently coloured colonies allowing rapid presumptive identification of different Candida species.

\section{Results and Discussion}

300 immunocompromised patients were included in the study and a total number of 368 samples were processed. In the immunocompromised group, HIV infected individuals constituted a large number $155(41.7 \%)$, compared to renal transplant recipients $40(10.8 \%)$, patients on immunosuppressive therapy $30(8 \%)$, patients with cancer $30(8 \%)$, diabetes mellitus $30(8 \%)$ and chronic disease 15(4\%). In our study Among the HIV infected patients, most of the samples were from oral cavity 62(38.5\%), followed by vaginal discharge 50(31\%), urine
$25(15.5 \%)$, sputum $10(6.2 \%)$, blood $8(4.9 \%)$ and CSF 6(3.7\%). Studies which have also reported the highest yield of Candida spp. from oral swabs and the lowest yield of Candida spp. from blood specimens from HIV positive patients (Wadhwa et al., 2007).

In renal transplant recipients, 40 samples were urine (61.5\%), 15 from oral cavity (20\%), 7 blood (10.7\%) and 3 CSF (4.6\%). Among the patients on immunosuppressive therapy which excludes the renal transplant recipients, 30 (60\%) samples were from oral scrapings, 10 (20\%) from urine, 8 (16\%) from sputum and 2 (4\%) from blood. 37 and 30 samples were obtained respectively from patients on cancer chemotherapy and patients with diabetesmainly from oral cavity and urine.

The most common species isolated was $C$. albicans (42\%), followed by $C$. tropicalis (35\%), C. pseudotropicalis (5\%), C. krusei (5\%), C. glabarata (4\%), C. parapsilosis (3\%), C. guilliermondii (2\%). Our study revealed Out of the 45 isolates of $C$. albicans, $34(75.5 \%)$ were from HIV patients followed by 5 in renal transplant recipients $(11 \%)$, $2(4.4 \%)$ each in patients on immunosuppressive therapy and on cancer chemotherapy and $1(2.2 \%)$ in a patient with diabetes. Of the 37 isolates of $C$. tropicalis, $11(29.7 \%)$ were from HIV infected patients, followed by $8(21.6 \%)$ from patients on immunosuppressive therapy, $4(10.8 \%)$ in diabetic patients, $3(8 \%)$ in patients with chronic diseases and 2(5.4\%) in patients on cancer chemotherapy. Among the $5 \mathrm{C}$. pseudotropicalis isolates, 3 were from patients with diabetes and 1 each from a patient with chronic disease. Out of 3 C. parapsilosis isolates, one isolate was obtained each from a HIV infected patient, patient on immunosuppressive therapy and a diabetic patient. C. krusei isolates were isolated 2 each from renal transplant recipients and diabetics and one from a patient on immunosuppressive therapy. Nweze et al., in their study showed 
though Candida albicans is the most parapsilosis, C. kefyr, and C. pelliculosa, have frequently isolated species other Candida become a significant cause of infection in species such as C. tropicalis, C. krusei, C. immunocompromised patients (Table 1-4). glabrata, C. dubliniensis, C. guilliermondii, $C$.

Table.1 Samples collected from patients

\begin{tabular}{|l|l|l|}
\hline Patient Category & No of patients & Total no of samples \\
\hline HIV & 155 & 161 \\
\hline Renal transplant Recipients & 40 & 65 \\
\hline Immunosuppressive therapy & 30 & 50 \\
\hline Cancer & 30 & 37 \\
\hline Diabetes & 30 & 30 \\
\hline Chronic disease & 15 & 17 \\
\hline Total & 300 & 368 \\
\hline
\end{tabular}

Table.2 Samples collected from patients

\begin{tabular}{|l|l|l|l|l|l|l|l|l|l|l|}
\hline Patient Category & Oral & Vaginal Urine & Blood & $\begin{array}{l}\text { Nasal } \\
\text { swab }\end{array}$ & $\begin{array}{l}\text { Tracheal } \\
\text { aspirate }\end{array}$ & Sputum & CSF & Palate & $\begin{array}{l}\text { Pyo } \\
\text { pneumo } \\
\text { thorax }\end{array}$ \\
\hline HIV & 62 & 50 & 25 & 8 & - & - & 10 & 6 & - & 6 \\
\hline $\begin{array}{l}\text { Renal transplant } \\
\text { Recipients }\end{array}$ & 15 & - & 40 & 7 & - & - & - & 3 & - & 3 \\
\hline $\begin{array}{l}\text { Immunosuppressive } \\
\text { therapy }\end{array}$ & 30 & - & 10 & 2 & - & - & 8 & - & - & - \\
\hline Cancer & 20 & - & 10 & 3 & - & - & 4 & - & - & - \\
\hline Diabetes & 10 & - & 10 & - & 3 & 4 & 3 & - & - & - \\
\hline Chronic disease & 3 & - & - & - & 4 & 4 & 6 & - & 1 & - \\
\hline
\end{tabular}

Table.3 Species of Candida isolated

\begin{tabular}{|c|c|c|c|c|c|c|c|c|c|}
\hline Patient Category & 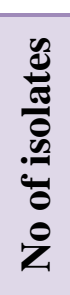 & 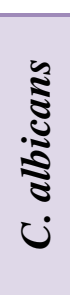 & 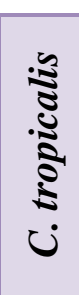 & ن & 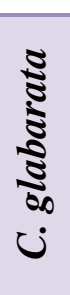 & 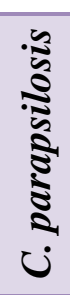 & $\frac{\widetilde{v}}{\tilde{5}}$ & 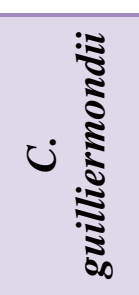 & 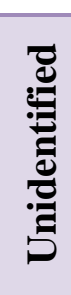 \\
\hline HIV & 50 & 34 & 11 & - & 1 & 1 & - & - & 3 \\
\hline $\begin{array}{l}\text { Renal transplant } \\
\text { Recipients }\end{array}$ & 12 & 5 & 2 & - & 3 & - & 2 & - & - \\
\hline $\begin{array}{l}\text { Immunosuppressive } \\
\text { therapy }\end{array}$ & 12 & 2 & 8 & - & - & 1 & 1 & - & - \\
\hline Cancer & 4 & 2 & 2 & - & - & - & - & - & - \\
\hline Diabetes & 11 & 1 & 4 & 3 & - & 1 & 2 & - & - \\
\hline Chronic disease & 4 & 0 & 3 & 1 & - & - & - & 2 & 3 \\
\hline
\end{tabular}


Table.4 Candida species in various clinical samples

\begin{tabular}{|c|c|c|c|c|c|c|c|c|}
\hline $\begin{array}{l}\text { Candida species in } \\
\text { Various Clinical } \\
\text { Samples species }\end{array}$ & $\overline{0}$ & 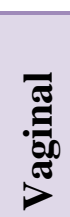 & 莡 & $\frac{\vec{\delta}}{\not \theta}$ & $\begin{array}{l}\bar{F} \\
\bar{c} \\
\bar{z} \\
\bar{z}\end{array}$ & 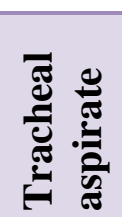 & 音 & $\frac{\text { ज̃ }}{\pi}$ \\
\hline C. albicans & 31 & - & 11 & - & - & 1 & 1 & 1 \\
\hline C. tropicalis & 14 & 4 & 9 & 1 & 2 & 2 & 3 & 1 \\
\hline C.pseudotropicalis & - & 1 & 2 & - & - & 2 & - & \\
\hline C. glabarata & 1 & - & 3 & - & - & 1 & - & \\
\hline C. parapsilosis & 1 & 1 & - & - & - & - & 1 & \\
\hline C. krusei & 1 & 1 & - & 2 & - & - & 1 & \\
\hline C. guilliermondii & - & 1 & - & - & - & - & 1 & \\
\hline Unidentified & 3 & - & - & - & - & - & 3 & \\
\hline
\end{tabular}

C. albicans was predominantly isolated from the oral cavity $31(68.8 \%)$ followed by urine $11(24.4 \%)$, bronchial wash $1(2.2 \%)$, sputum $1(2.2 \%)$ from palatal perforation. Majority of the $C$. tropicalis isolates were obtained from oral cavity $14(37.8 \%)$ followed by urine $9(24.3 \%)$, vagina $4(10.8 \%)$, sputum $3(8.1 \%)$ and $2(5.4 \%)$ each from nasal swab, tracheal aspirate and bronchial wash. $C$. pseudotropicalis was isolated $2(40 \%)$ each from urine and tracheal aspirate and one (205) from vagina. C. glabrata was isolated more from urine $3(75 \%)$ than from oral cavity 1 (25\%). 3 isolated of $C$. parapsilosis were obtained one each from oral cavity, vaginal discharge and sputum. C. krusei isolates were obtained 2(40\%) from blood and one each from oral cavity, vaginal discharge and sputum. Monika Maheshwari et al., had a similar observation in their study. Among $C$. albicans strains, the maximum number (was from oral swabs followed by sputum and urine (6/64), while most of C. glabrata strains were isolated from urine samples. $C$. dubliniensis, C. parapsilosis, C. krusei, $C$. tropicalis, and C. kefyr were mainly detected in oral swabs.

In conclusion, along with Candida albicans, non albicans Candida spp like C. tropicalis,
C, krusei and C. glabrata are increasingly being isolated from clinical specimen among immunocompromised patients. CHROM agar Candida not only facilitates the detection of mixed cultures but also allows for a presumptive identification to the species level so as to help the clinicians to choose appropriate antifungal agents.

\section{References}

Gugnani, H.C., Becker. K., Fegeler et al., "Oropharyngeal carriage of Candida species in HIV-infected patients in India," Mycoses, 2003 vol. 46, no. 8, pp. 299-306.

Lata R Patel, Jayshri D Pethani, Palak Bhatia, Sanjay D Rathod, Parul D Shah. Prevalence of Candida infection and its antifungal susceptibility pattern in Tertiary Care Hospital, Ahmedabad. National Journal of Medical Research Oct - Dec 2012Volume 2 | Issue 4 |

Manchanda V., Agarwal S., Verma N. Yeast identification in routine clinical Microbiology laboratory and its clinical relevance. Indian Journal of Medical Microbiology 2011; 29(2):172

Monika Maheshwari, Ravinder Kaur, and Sanjim Chadha Candida Species 
Prevalence Profile in HIV Seropositive Patients from a Major Tertiary Care Hospital in New Delhi, India Hindawi Publishing Corporation Journal of Pathogens Volume 2016, Article ID 6204804, 8 Pp.

Nweze E.I and Ogbonnaya, U.L "Oral Candida isolates among HIV-infected subjects in Nigeria," Journal of
Microbiology, Immunology and Infection, 2011 vol. 44, no. 3, pp. 172177

Wadhwa A, Kaur R, Agarwal, S.K S. Jain, and P. Bhalla, "AIDS related opportunistic mycoses seen in a tertiary care hospital inNorth India," Journal of Medical Microbiology, vol. 56, part 8, pp. 1101-1106, 2007.

\section{How to cite this article:}

Meenakshi, S. and Vidhya Rani, R. 2019. Speciation of Candida Isolated among Immunocompromised Patients. Int.J.Curr.Microbiol.App.Sci. 8(03): 1582-1586.

doi: https://doi.org/10.20546/ijcmas.2019.803.183 